\title{
Cholecystectomy with Thoracic Epidural Anesthesia in Geriatric Patients
}

\author{
Sedat Saylan, Ali Akdogan, Davut Dohman and Erdem Nail Duman \\ Department of Anesthesiology and Intensive Care Medicine, Faculty of Medicine, Karadeniz Technical University, Hospital, Trabzon, \\ Turkey
}

\begin{abstract}
Objective: To determine the outcome of thoracic epidural anaesthesia in open cholecystectomy procedures of comorbid advanced elderly patients.

Study Design: Observational study.

Place and Duration of Study: Faculty of Medicine, Karadeniz Technical University Hospital, Trabzon, Turkey during 2014-2019.

Methodology: This study included 103 geriatric patients of over 65 years, who underwent open cholecystectomy under thoracic epidural anaesthesia during the study period. Patient files, anaesthesia registration forms, early postoperative followup, and discharge process were retrospective.

Results: The mean age of the patients included in the study was $79.42 \pm 9.03$ years, while the mean operation time was 68.02 \pm 17.44 minutes. While only $7(6.8 \%)$ patients had a chronic disease, the number of patients with two or three comorbid diseases was $67(65 \%)$. The number of patients with 4 or more comorbidities was $29(28.2 \%)$. The most common intraoperative complications were hypotension $(n=22,21.4 \%)$ and bradycardia $(n=19,18.4 \%)$. Intraoperative desaturation was observed in 7 (6.8\%) patients; and oxygen and bronchodilator therapy was effective. The median discharge time was 6.5 (5.0-8.25) days; whereas, 5 (4.9\%) patients died postoperatively.

Conclusion: In open cholecystectomy operations on comorbid advanced elderly patients, thoracic epidural anaesthesia can be preferred to general anaesthesia.
\end{abstract}

Key Words: Geriatrics, Cholecystectomy, Anesthesia, Thoracic epidural, Comorbidity.

How to cite this article: Saylan S, Akdogan A, Dohman D, Duman EN. Cholecystectomy with Thoracic Epidural Anesthesia in Geriatric Patients. J Coll Physicians Surg Pak 2021; 31(04):445-449.

\section{INTRODUCTION}

Compared with young adults, geriatric patients undergoing surgery may have various negative factors, such as decreased physiological capacities, comorbid diseases, multiple drug use, cognitive dysfunction, and fragility; which may increase morbidity and mortality due to complications associated with anesthesia. ${ }^{1}$ In these patients, the ideal approach during surgical or anaesthetic procedures is to keep the hemodynamic values stable and minimise complications. Comorbid diseases can also be seen frequently in these patients, which makes the choice of the anaesthesia method crucial.

Correspondence to: Dr. Sedat Saylan, Department of Anes-

thesiology and Intensive Care Medicine, Faculty of

Medicine, Karadeniz Technical University, Hospital,

Trabzon, Turkey

E-mail: sedatsaylan@yahoo.com

Received: August 19, 2020; Revised: October 18, 2020;

Accepted: December 08, 2020

DOI: https://doi.org/10.29271/jcpsp.2021.04.445
Most clinical trials comparing regional anaesthesia (RA) and general anaesthesia (GA) in the elderly have not demonstrated the superiority of one technique over the other. However, RA allows patients to maintain their own airway safety and respiratory function without requiring airway intervention. ${ }^{2,3}$

In abdominal surgery, epidural anaesthesia/analgesia reduces complications of GA, such as mechanical ventilation, myocardialdepression, and prolonged ileus, whilepreserving cardiopulmonary functions. Traditionally, GA is used for open cholecystectomy (OC), but recently, RA techniques have also been used successfully. ${ }^{3}$

The objective of the present study was to describe the outcome of thoracic epidural anaesthesia (TEA) in OC for comorbid advanced elderly patients.

\section{METHODOLOGY}

The local Ethics Committee approved this observational study (approval No. 2019/128). This study included 105 geriatric patients of $>65$ years of age, who underwent OC at Faculty of Medicine, Karadeniz Technical University Hospital, Trabzon, Turkey, under TEA between 2014 and 2019 (Figure 1). The 
patients who were $<65$ years of age and who underwent laparoscopic cholecystectomy under general anaesthesia were excluded from the study.

To evaluate the effectiveness of TEA, patient files, anaesthesia registration forms, early postoperative follow-up, and discharge process were retrospectively analysed. The information collected from the medical records included demographic data, American Society of Anaesthesiologists (ASA) class, comorbid diseases, complications such as bradycardia, hypotension, hypertension, arrhythmia, and desaturation, and medications with doses. The intervertebral level of the TEA intervention, local anaesthetics used, duration of the intervention, and local anaesthetics applied from the epidural catheter after surgery were also noted. Patients who were transferred to GA for any reason or developed intraoperative surgical complications were excluded from the study.

Informed patient consent form was obtained routinely before each procedure. TEA was performed under all aseptic and antiseptic measures in the sitting or in the lateral decubitus position. ECG, non-invasive blood pressure measurement, and pulse-oximeter monitoring were performed in all patients before the procedure. After administering local anaesthesia, the epidural space was entered at the T7-T10 level using an 18gage Tuohy needle with the hanging drop method. The test dose was made by giving $3 \mathrm{~mL}$ of $2 \%$ lidocaine without adrenaline from the epidural catheter; a 5-min wait time was applied to ensure that it was not in the subarachnoid range. The dose protocol was a $20 \mathrm{~mL}$ mixture of $0.5 \%$ bupivacaine $(10 \mathrm{~mL}), 2 \%$ lidocaine (4 mL), fentanyl $50 \mathrm{mcg}(1 \mathrm{~mL})$, and isotonic saline (5 $\mathrm{mL}$ ). A bolus $5 \mathrm{~mL}$ dose of this mixture was administered. If the hemodynamic response was stable after the bolus dose, 5-mL divided doses were given at 5-10-min intervals until the sensory block level reached the T4 dermatome. Pin-prick test was used for block-level control, atropine was administered in case of bradycardia (defined as heart rate $<50$ beats/min); and fluid support and ephedrine were given in case of hypotension. Hypotension was defined as a decrease in systolic and diastolic arterial pressures by $25 \%$ compared with baseline value or systolic arterial pressure $<90 \mathrm{mmHg}$, and 5-10 mg ephedrine was administered intravenously (IV). Oxygen support 2-4 L/min was provided to all our patients through a face mask with capnographymonitoring.

The data were analysed using SPSS version 23.0 (SPSS, Chicago, IL). The normal distribution of numerical variables was evaluated with the Kolmogorov-Smirnov test. Descriptive statistics were drawn and numerical variables were presented as mean \pm standard deviation and median (IQR: 25\%-75\%) and categorical variables as number (n) and percentage (\%).

\section{RESULTS}

Demographic data, preoperative features, risk factors and basic parametric values of the patients are shown in Tablel.

Two of 105 patients, who underwent TEA, were excluded from the study because GA had to be applied as a result of intraopera- tive surgical complications. Comorbid diseases of 103 patients included in the study are given in Table I. Only 7 (6.8\%) patients had one chronic disease, and 67 (65\%) and 29 (28.2\%) patients had 2-3and $>4$ comorbid diseases, respectively.

Table I: Demographic and main parametric data, comorbid diseases, intraoperative complications and medicines used.

\begin{tabular}{|l|l|}
\hline Demographic and main & $\mathbf{n}=\mathbf{1 0 3}$ \\
parametric data & $79.42 \pm 9.03$ \\
\hline Age [year, (mean \pm SD)] & $58(56.3 \%)$ / $45(43.7 \%)$ \\
Gender [female/ male, $\mathrm{n}(\%)]$ & $26.08 \pm 2.86$ \\
Body Mass Index (kg/m $\left.{ }^{2}\right)$ & $11(10.7 \%) / 73(70.9 \%) /$ \\
ASA $^{1}$ Physical Status (2/3/4) & $19(18.4 \%)$ \\
Duration of surgery (minute) & $68.02 \pm 17.44$ \\
Total intravenous fluid (crystalloid, & $799.03 \pm 425.06$ \\
mL) & \\
Discharge time (days) [Median (IQR: & $6.5(5.0-8.25)$ \\
25\%-75\%)] & $5(4.9 \%)$ \\
Patients with exitus & $\mathrm{n}(\%)$ \\
\hline Comorbid diseases & $96(93.2 \%)$ \\
\hline Hypertension & $46(44.7 \%)$ \\
Rhythm disturbances & $42(40.8 \%)$ \\
Congestive heart failure & $33(32.0 \%)$ \\
Diabetes mellitus & $31(30.1 \%)$ \\
Coronary artery disease & $27(26.2 \%)$ \\
Asthma bronchiole & $23(22.3 \%)$ \\
Chronic obstructive pulmonary disease & $7(6.8 \%)$ \\
Chronic kidney disease & $4(3.9 \%)$ \\
Acute kidney failure & $4(3.9 \%)$ \\
Alzheimer's disease & $2(1.9 \%)$ \\
Epilepsy & $\mathrm{n}(\%)$ \\
\hline Intraoperative complications and & $22(21.4 \%)$ \\
medicines used & $19(18.4 \%)$ \\
\hline Hypotension & $17(16.5 \%)$ \\
Sinus bradycardia & $7(6.8 \%)$ \\
Rhythm disturbances & $2(1.9 \%)$ \\
Desaturation & $1(1.0 \%)$ \\
Hypertension & $12.89 \pm 5.09$ \\
Sinus tachycardia & $0.63 \pm 0.23$ \\
Ephedrine requirement (mg) & \\
Atropine requirement (mg) & \\
\hline${ }^{1}$ ASA: American Society of Anesthesiologists. \\
\hline
\end{tabular}

Intraoperative complications of patients, and the most frequently used drugs are shown in Table I. Although hypotension ( $n=22,21.4 \%$ ) and bradycardia ( $n=19,18.4 \%)$ developed in some of the patients, hemodynamic stability was achieved without any complications with atropine, ephedrine and fluid therapy. Perioperative mean blood pressure and mean heart rate changes of our patients a re given in Figure 2.

Midazolam (1.70 $\pm 0.71 \mathrm{mg})$, fentanyl (40.47 $\pm 10.47 \mathrm{mcg})$ and propofol ( $33.33 \pm 8.17 \mathrm{mg}$ ) were used mostly for the purpose of sedoanalgesia. Postoperative nausea and vomiting (PONV) developed in $19(18.4 \%)$ of patients and were treated with IV ondansetron. The mean discharge time of the patients was 6.5 (5.0-8.25) days. Five (4.9\%) patients died postoperatively: one of them had ASA class III risk and the others had ASA class IV, and all of them had multiple comorbid diseases. Two patients died from acute myocardial infarction on the 2 nd and 4 th days, and one patient each from pneumonia on the $13^{\text {th }}$ day, pulmonary embolism on the 3rd day, and sepsis due to surgical site infection on the 11th day. 


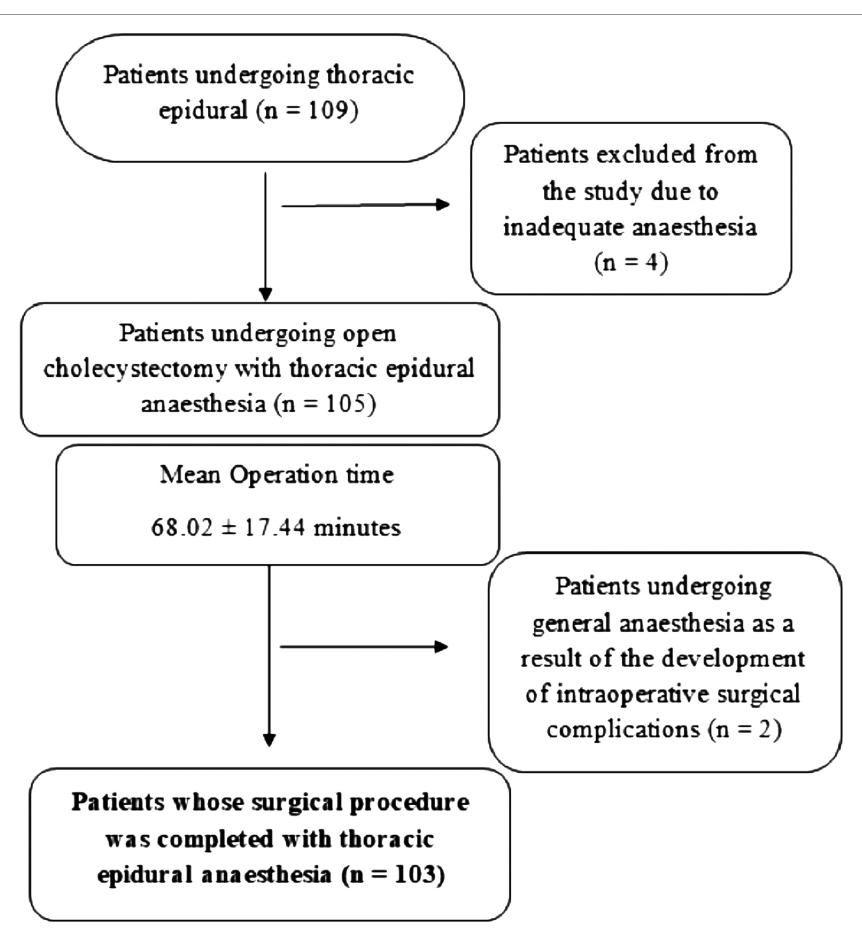

Figure 1: Study flow diagram.

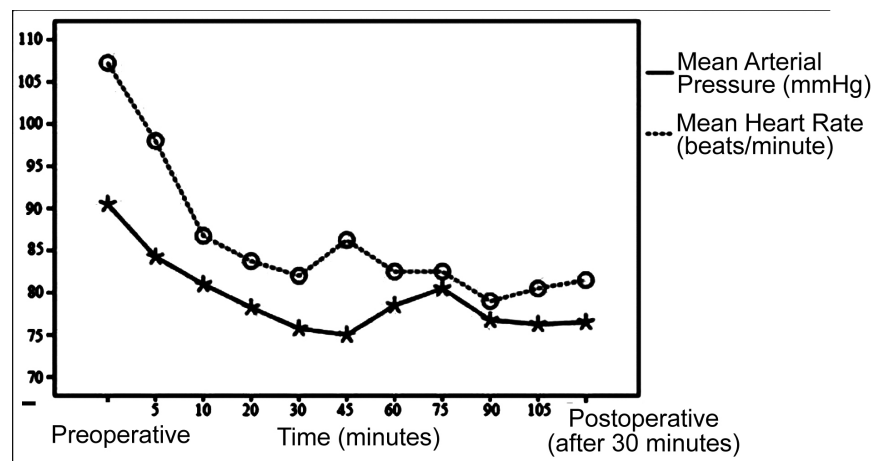

Figure 2: Mean arterial pressure ( $\mathrm{mmHg}$ ) and mean heart rate (bets/minute).

\section{DISCUSSION}

Aging is associated with a decline in physiological reserves, especially cardiovascular, respiratory, neurological, and kidney functions. These lead to changes in the metabolism of drugs, necessitating caution in drug use and adjustment of drug doses. The ideal anaesthetic approach in the elderly is to be the least invasive, thereby minimising changes in homeostasis. Most clinical trials, comparing RA and GA in the elderly, found comparable. ${ }^{2}$

Arslan et al. found no difference in the complication rates between young and older patients undergoing OC, if appropriate surgical preparations were made. However, they stated that the duration of hospitalisation and return to daily activities in comorbid older patients with a high ASA class was significantly longer than that in younger patients. ${ }^{4}$ All of the patients in this study had at least one comorbid disease. While most of the present patients had ASA class III ( $n=73,70.9 \%), 19(18.4 \%)$ patients had ASA class IV.

The sensory block level must be at least above the T6 level to perform laparoscopic cholecystectomy (LC) underTEA. In addition, the combination of fentanyl with local anaesthetics prolongs the sensory block in the central neuraxial blockade. ${ }^{5}$ Sunamak et al. indicated that combined spinal-epidural anaesthesia (CSEA) is effective and feasible for LC in ASA class III patients, obese patients, and those with comorbidities. Although the sensory block level above T6 is sufficient for spinal anaesthesia (SA) or for LC under TEA, the optimal block level remains unclear. Sunamak et al. achieved sensory block at the T4 level without serious side effects related to CSEA. ${ }^{6}$ In these patients, the surgical procedure was allowed after the sensory block level reached to T4 level following TEA.

In EA, block-level titration is easier, and it provides a good analgesia without causing motor block. Therefore, EA in which the sympathetic block starts more slowly may be preferred, especially in patients with cardiopulmonary disease. ${ }^{7}$ A local anaesthetic can be given at once or in divided doses. The incidence of hypotension decreases when the doses are divided, with fewer and delayed early complications, thus affording time for intervention. ${ }^{8}$ Rapidly developing sympathetic block with a single dose of local anaesthetic may not be easily or quickly compensated in patients with decreased cardiac reserve due to aging or disease, leading to severe consequences. Arık et al. concluded that the application of divided doses of the local anaesthetic in EA in elderly and cardiac-risk patients is hemodynamically more stable. ${ }^{9}$ At the study centre, medications are administered in comorbid geriatric patients with frequent hemodynamic monitoring and in divided doses via the epidural catheter.

The reduction in functional residual capacity in patients undergoing GA is higher than in RA. With RA, respiratory functions are not or minimally affected; thus, carbon dioxide can be eliminated more easily. ${ }^{10}$ RA may also have some advantages over GA, such as prevention of mouth and teeth injuries, sore throat, and stomach swelling that may occur due to laryngoscopy and mask ventilation with GA. Furthermore, patients are awake and conscious at the end of the operation and experience less postoperative pain, nausea, and vomiting. Zhang et al. retrospectively analysed 100 patients undergoing LC under EA and found EA to have a similar safety profile as GA for patients undergoing LC. ${ }^{11}$

RA is an acceptable technique in patients with ischemic heart disease. However, hypotension associated with EA or SA should be treated quickly. Potential benefits of RA in cardiac patients include excellent pain control, decreased neuroendocrine response, decreased incidence of deep vein thrombosis, and the opportunity to provide postoperative analgesia. ${ }^{12}$ TEA combined with GA can suppress the stress reaction and provide pain control and hemodynamic stabilization in the first 24-hour intraoperatively and postoperatively. ${ }^{13}$ Although some of the patients developed hypotension ( $n=22,21.4 \%$ ) and bradycardia ( $n=19,18.4 \%$ ) after EA, hemodynamic stability was achieved with atropine, ephedrine, and fluid treatment without any complications. 
The goals of anaesthetic management in lung diseases, such as chronic obstructive lung disease (COPD) and bronchial asthma, should be avoiding drugs that cause respiratory depression and cause mucociliary clearance disorder and avoiding mechanical ventilation. Therefore, good analgesia and early mobilisation are critical to prevent the worsening of respiratory mechanics, especially in upper abdominal surgeries. Compared with GA, EA reduces respiratory complications such as pneumonia, atelectasis, and hypoxemia in patients at risk of pulmonary complications, resulting in faster recovery, lower postoperative pain, and better cost-benefit ratio. ${ }^{14}$ Gramatica et al. evaluated patients with COPD, who underwent LC with TEA, and concluded that TEA is a viable option in patients who pose a risk for GA due to cardiopulmonary and airway problems, reducing the need for postoperative analgesia and facilitating quick and smooth recovery. ${ }^{15}$ Likewise, Bilgi et al. emphasised that TEA may be preferred to GA in LC surgeries because TEA affects respiratory functions less, causes fewer postoperative complications, and provides more effective postoperative analgesia. ${ }^{16}$ In this study, $27(26.2 \%)$ patients had asthma and $23(22.3 \%)$ had COPD, but only $7(6.8 \%)$ patients developed intraoperative desaturation and all responded to $\mathrm{O}_{2}$ and bronchodilator therapy, achieving spontaneous breathing and adequate peripheral $\mathrm{O}_{2}$ saturation values.

Donmez et al. compared CSEA and GA in patients undergoing LC and found CSEA to be suitable, sufficient, and safe for LC, with a comparatively lower incidence of postoperative pain, shoulder pain, and PONV. ${ }^{17}$ Intraoperative adverse events associated with CSEA can be treated easily. CSEA may thus be an alternative to GA in patients at high risk or contraindication for GA. Donmez et al. treated PONV effectively with IV $8 \mathrm{mg}$ ondansetron. ${ }^{17}$ In this study, PONV developed in 19 (18.4\%) patients and was treated with IV 4-8 mg ondansetron.

RA does not require airway instrumentation and allows patients to maintain their own airway and respiratory function levels, with a lower risk of hypoxemia.

However, the present study has some limitations. The authors did not include a control group of patients undergoing EA with GA or only GA. Moreover, blood gas analysis, pulmonary function tests, postoperative cognitive evaluations, and follow-up of vital parameters or organ dysfunctions were not evaluated, which could be examined in future studies. In addition, the prolongation of the discharge time was not investigated.

\section{CONCLUSION}

TEA can be preferred to GA in high-risk comorbid geriatric patients undergoing $\mathrm{OC}$ by blocking the desired sensory level effectively and providing more stable hemodynamics without airway manipulation.

\section{ETHICALAPPROVAL:}

Ethics Committee approval was received for this study from the Ethics Committee of Faculty of Medicine, Karadeniz Technical University, Ethics Review Committee (2019/128).

\section{PATIENTS' CONSENT:}

Not applicable as this is a retrospective study. Moreover, patient identifiers were removed before data analysis for confidentiality.

\section{CONFLICT OF INTEREST:}

Authors declared no conflict of interest.

\section{AUTHORS' CONTRIBUTION:}

SS, AA, DD, END: Design, supervision, data collection and/or processing, analysis and/or interpretation, literature review, and critical review.

\section{REFERENCES}

1. Griffiths R, Beech F, Brown A, Dhesi J, Foo I, Goodall J, et al. Peri-operative care of the elderly 2014: Association of Anaesthetists of Great Britain and Ireland. Anaesthesia. 2014; 69(Suppl 1):81-98. doi: 10.1111/anae.12524.

2. Le-Wendling L, Bihorac A, Baslanti TO, Lucas S, Sadasivan $K$, Wendling $A$, et al. Regional anesthesia as compared with general anesthesia for surgery in geriatric patients with hip fracture: does it decrease morbidity, mortality, and health care costs? Results of a single-centred study. Pain Med 2012; 13:948-56. doi: 10.1111/j.1526-4637.2012.01402.x.

3. Mehta N, Gupta S, Sharma A, Dar MR. Thoracic combined spinal epidural anesthesia for laparoscopic cholecystectomy in a geriatric patient with ischemic heart disease and renal insufficiency. Local Reg Anesth 2015; 8:101-04. doi: 10.2147/LRA.S86390.

4. Arslan B, Yılmaz T, Erol V, Tuncalı B, Gülay H. Can cholecystectomy be performed as safely in elderly patients as it is in young patients? turkish journal of geriatrics 2020; 23(1):8-17. doi: 10.31086/tjgeri.2020.132.

5. Imbelloni LE. Spinal anesthesia for laparoscopic cholecystectomy: Thoracic vs. Lumbar Technique. Saudi J Anaesth 2014; 8:477-83. doi: 10.4103/1658-354X.140853.

6. Sunamak O, Donmez T, Uzman S, Erdem VM, Erdem DA, Yıldırım D, et al. Laparoscopic Cholecystectomy Under Combined Spinal/Epidural Anesthesia: A retrospective analysis of 112 cases in terms of per- and postoperative outcomes. Haydarpasa Numune Med J 2018; 58(1):5-11. doi: 10.14744/hnhj.2017.60783.

7. Lee HJ, Huh J, Kim DK, Gil JR, Min SW, Han SS. Laparoscopic cholecystectomy under epidural anesthesia: a clinical feasibility study. Korean J Anesthesiol 2010; 59(6):383-8. doi: 10.4097/kjae.2010.59.6.383.

8. Karinen J, Makarainen L, Alahuhta S, Jouppila R, Jouppila P. Single bolus compared with a fractionated dose injection technique of bupivacaine for extradural Caesarean section: effect on uteroplacental and fetal haemodynamic state. $\mathrm{Br} J$ Anaesth 1996; 77(2):140-4. doi: 10.1093/bja/77.2.140.

9. Arık H, Erhan OL, Beştaş A, Özer AB, Yıldızhan Ö. The effect of a single or fractional dose of local anesthetic on hemodynamics in epidural anesthesia evaluated according to ejection fraction. Turk J Geriatr 2012; 15:439-44.

10. Bablekos GD, Michaelides SA, Analitis A, Charalabopoulos KA. Effects of laparoscopic cholecystectomy on lung function: A systematic review. World J Gastroenterol 2014; 20:17603-17. doi: 10.3748/wjg.v20.i46.17603. 
11. Zhang HW, Chen YJ, Cao MH, Ji FT. Laparoscopic cholecystectomy under epidural anesthesia: A retrospective comparison of 100 patients. Am Surg 2012; 78:107-10. PMID: 22273326.

12. Liu SS, Wu CL. Effect of postoperative analgesia on major postoperative complications: A systemic update of the evidence. Anesthesia \& Analgesia 2007; 104(3):689-702. doi: 10.1213/01.ane.0000255040.71600.41.

13. Ozcan S, Ozer AB, Yasar MA, Erhan OL. Effects of combined general anesthesia and thoracic epidural analgesia on cytokine response in patients undergoing laparoscopic cholecystectomy. Niger J Clin Pract 2016; 19(4):436-42. doi: 10.4103/1119-3077.183308.

14. Grass JA. The role of epidural anesthesia and analgesia in postoperative outcome. Anesthesiol Clin North Am 2000; 18(2):407-28. doi: 10.1016/s0889-8537(05)70170-x.
15. Gramatica Jr L, Brasesco OE, Mercado AL, Martinessi V, Panebianco G, Labaque $F$, et al. Laparoscopic cholecystectomy performed under regional anesthesia in patients with chronic obstructive pulmonary disease. Surg Endosc 2002; 16(3):472-5. doi: 10.1007/s00464-0018148-0.

16. Bilgi M, Alshair EE, Göksu H, Sevim O. Experience of Laparoscopic Cholecystectomy Under Thoracic Epidural Anaesthesia: Retrospective Analysis of 96 Patients. Turk J Anaesthesiol Reanim 2015; 43(1):29-34. doi: 10.5152/ TJAR.2014.68926.

17. Donmez T, Erdem VM, Uzman S, Yildirim D, Avaroglu HI, Ferahman S, et al. Laparoscopic cholecystectomy under spinal-epidural anesthesia vs. general anaesthesia: a prospective randomised study. Ann Surg Treat Res 2017; 92(3):136-142. doi: 10.4174/astr.2017.92.3.136. 